\title{
REVIEWER LIST 1995
}

We extend our sincerest gratitude to the following reviewers who have contributed their time and expertise to our journal. This list includes all individuals who provided or delegated reviews for papers appearing in Volume 3, Issues 1 through 4, of the Journal of Intelligent \& Fuzzy Systems.

\section{Mo Jamshidi and Timothy J. Ross, Editors-in-Chief} Valerie Steinmaus, Editorial Manager

\author{
Teresa Adams \\ University of Wisconsin at Madison \\ Mohammad Akbarzadeh \\ University of New Mexico \\ Jack Aldrich \\ AdvanTex, Inc. \\ M. Anvari \\ University of California, Berkeley \\ Adedeji B. Badiru \\ University of Oklahoma \\ Denis Barak \\ Control Designs, New Mexico \\ Senen Barro-Ameneiro \\ Universidade de Santiago de Compostela \\ R. Bertin \\ GRAI-Laboratoire d'Automatique et Productique \\ Doug Birdwell \\ University of Tennessee, Knoxville \\ B. Bourgeois \\ University of Houston \\ A. Bugarin \\ Universidade de Santiago de Compostela \\ Deqian Chen \\ University of Arizona \\ Stephen Chiu \\ Rockwell Science Center \\ Byeong-Mook Chung \\ Korea Advanced Institute of Science \& Technology \\ (KAIST) \\ R. Colbaugh \\ New Mexico State University \\ George Cunningham III \\ New Mexico Institute of Mining \& Technology \\ Ahmed M. Darwish \\ Cairo University \\ M. Delgado-Restituto \\ Universidad de Sevilla, Spain \\ Kofe Kissi Dompere \\ Howard University \\ Didier Dubois \\ Universite Paul Sabatier, France \\ D. Edwards \\ University of Idaho \\ A.A. El-Keib \\ The University of Alabama
}

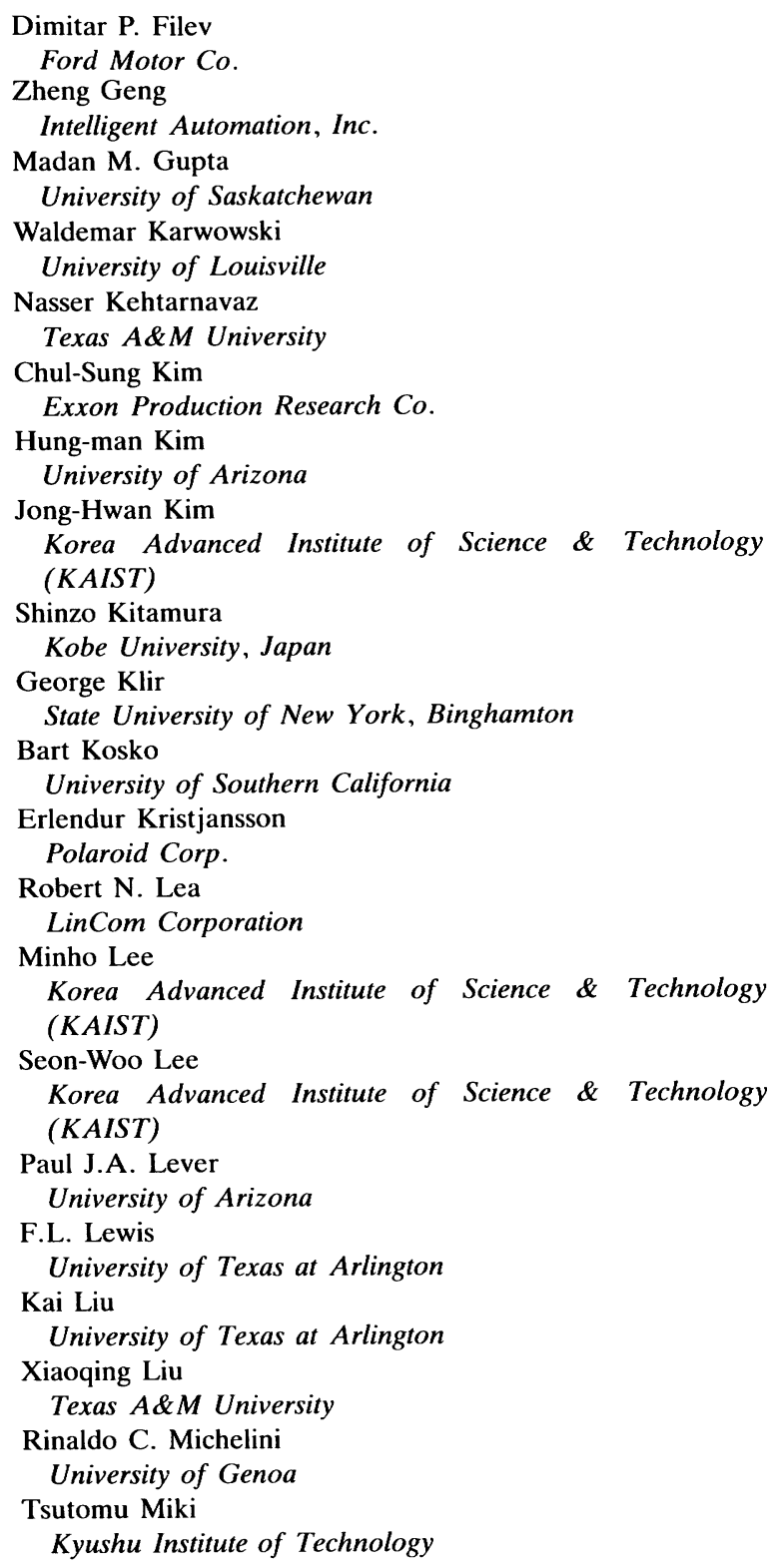


Gary P. Moynihan

The University of Alabama

M.H. Nehrir

Montana State University

H.T. Nguyen

New Mexico State University

Nikhil R. Pal

Indian Statistical Institute

Grantham Pang

Hitachi Research Laboratory, Japan

Cheol Hoon Park

Korea Advanced Institute of Science \& Technology (KAIST)

William J. Parkinson

Los Alamos National Laboratory

Bozenna Pasik-Duncan

University of Kansas

A. Patra

Indian Institute of Technology

Witold Pedrycz

University of Manitoba

Francois Pin

Oak Ridge National Laboratory

Alan Pugh

University of Hull, U.K.

P.K. Rajan

Tennessee Technical University

Ganti Prasada Rao

Indian Institute of Technology

Utpal Roy

Syracuse University

R. Ruiz

Universidade de Murcia

Nader Sadegh

Georgia Institute of Technology

Heloisa Scarpelli

UFS Car/CCT/DC, Brasil

Nariman Sepehri

University of Manitoba

Adnan K. Shaout

University of Michigan
Xiaobo Shi

University of Arizona

Divyendu Sinha

City University of New York

Qiang Song

University of Alabama

Harald Stadlbauer

Technical University of Vienna

Thomas Sudkamp

Wright State University

Hiroyuki Tamura

Osaka University, Japan

G.E. Taylor

University of Hull, U.K.

George Vachtsevanos

Georgia Institute of Technology

Nader Vadiee

University of New Mexico

S.J. Verzi

University of New Mexico

Claude Vibet

Institut Universitaire de Technologie

Miomir K. Vukobratovich

Mihailo Pupin Institute, Serbia

Feiyue Wang

University of Arizona

Paul P. Wang

Duke University

Jonathan Weiss

McDonnell Douglas Advanced Software Technology

Ronald R. Yager

Machine Intelligence Institute, Iona College

Hong Quan Yang

Simon Fraser University

Lianwen Zhang

University of British Columbia

Meng Chu Zhou

New Jersey Institute of Technology

A. Zilouchian

Florida Atlantic University 